\title{
A Clinical and Pathological Study of Kikuchi- Fujimoto Disease in A University Hospital in Saudi Arabia
}

\author{
Fahad A. Alghamdi', MBBS, Mohammed S. Abdelwahed ${ }^{2,3}$, PhD, \\ Abdulaziz Alghamdi ${ }^{4}$, STUDENT, Hani Z. Marzouki ${ }^{5}$, MBBS, \\ Ghadeer A. Mokhtar ${ }^{1}$, MBBS, and Shadi S. Alkhayyat ${ }^{6}$, MBBS \\ ${ }^{1}$ Department of Pathology, Faculty of Medicine, King Abdulaziz University, \\ Jeddah, Saudi Arabia \\ ${ }^{2}$ Department of Pathology, Faculty of Medicine, University of Jeddah, Jeddah, Saudi Arabia \\ ${ }^{3}$ Department of Pathology, Faculty of Medicine, Alazhar University, Cairo, Egypt \\ ${ }^{4}$ Faculty of Dentistry, King Abdulaziz University, Jeddah, Saudi Arabia \\ ${ }^{5}$ Department of Otolaryngology - Head and Neck Surgery, Faculty of Medicine, King Abdulaziz University, \\ Jeddah, Saudi Arabia \\ ${ }^{6}$ Department of Medicine, Faculty of Medicine, King Abdulaziz University, Jeddah, Saudi Arabia
}

\section{Correspondence}

Dr. Fahad A. Alghamdi

P.O. Box 8316

Jeddah 23423-3467

e.M: alalghamdi@kau.edu.sa

\section{Submission: 19 Feb 2018}

Accepted: $\quad 05$ Mar 2018

\section{Citation}

Alghamdi FA, Abdelwahed MS, Alghamdi A, Marzouki HZ, Mokhtar GA, Alkhayyat SS. A clinical and pathological study of KikuchiFujimoto disease in a university hospital in Saudi Arabia . JKAU Med Sci 2018; 25 (1): 23-29. DOI: 10.4197/Med. 25.1.4

\begin{abstract}
Kikuchi-Fujimoto disease is a benign disease of self-limiting course characterized by lymphadenopathy that is often mistaken clinically as lymphoma or infectious lymphadenitis. To prevent such a mistake, a clinical and pathological correlation is recommended. In this retrospective study, we are documenting the clinical and pathological features of this disease through the last 15 years (2001-2016) in King Abdulaziz University Hospital; and comparing results with that of international findings. From a total number of 1805 lymph nodes sent for histopathological examination during that period, only 9 cases had been diagnosed as Kikuchi-Fujimoto disease. Three (33\%) cases were 20 years old and under; five (56\%) cases were in the ages between 20 to 40 years old and only one (11\%) was above 40 years old. Eight (89\%) cases were females. Regarding the outcome, all cases showed complete recovery and there was no recurrence. Only two histological phases were seen, namely proliferative and necrotizing phases.
\end{abstract}

\section{Keywords}

Kikuchi-Fujimoto disease; Histiocytic necrotizing lymphadenitis

\section{Introduction}

ikuchi-Fujimoto disease (KFD; or histiocytic necrotizing lymphadenitis) is a disease of benign self-limiting course, affecting women more than men at a young adult age ${ }^{[1]}$. There is no definite cause yet identified; although some link between KFD and a few viruses and some autoimmune diseases like systemic lupus erythematosus has been proposed ${ }^{[2]}$.

Although it is a benign disease, the clinical presentations are often not straightforward while 
the differential diagnosis is broad including tuberculosis, lymphoma, and some connective tissue diseases ${ }^{[2]}$. Patients present usually with unilateral lymphadenopathy, more commonly at the neck region. It could sometimes be painful and in few occasions, it can be preceded by upper respiratory tract infection. Other less common clinical presentations include fever, enlarged spleen and parotid gland, skin rash, arthralgia, muscle ache, meningitis (aseptic) and bone marrow hemophagocytosis ${ }^{[1]}$. Laboratory findings are usually nonspecific. Mild neutropenia, high erythrocyte sedimentation rate, and lymphocytosis were seen in some cases $^{[3]}$.

On the histological point of view, the disease usually affects the nodal cortical and paracortical areas ${ }^{[4]} . \mathrm{Kuo}^{[5]}$ has described three distinct histological phases: proliferative, necrotizing, and xanthomatous. In the proliferative phase, the involved nodal areas show proliferative foci composed of plasmacytoid monocytes, small- to medium-sized lymphocytes, and immunoblasts (mostly CD3+/CD8+) associated with some karyorrhectic apoptotic debris. Later, foci of paracortical nodal coagulative necrosis will appear (necrotizing phase). Xanthomatous phase is characterized by many foamy histiocytes $s^{[4,5]}$.

Due to the presence of numerous amounts of immunoblasts in early lesions without overt necrosis, these lesions can be misdiagnosed as malignant lymphoma ${ }^{[4]}$. However, immunohistochemistry study is helpful to exclude malignant lymphoma in those difficult cases. In cases of KFD, immunoblast cells are usually positive for $T$ cell markers, mostly CD8+; while histiocytes express CD68 antigens and myeloperoxidase ${ }^{[5]}$.

The current study is conducted by examination of lymph node tissues seen through the last 15 years (2001-2016) in King Abdulaziz University Hospital to show how many of them were Kikuchi disease and to review the age and sex ratio, clinical presentation and outcome, pathological features in those patients and compare results with that of international literature findings.

\section{Patients and Methods}

This was a retrospective study of cases of KFD diagnosed between 2001 and 2016 at King Abdulaziz University Hospital. The cases were collected from the archives using the coding system and databases of the hospital.

First, all cases which underwent surgical biopsy of lymph nodes were calculated. Then the cases diagnosed as KFD were determined and the percentage was calculated.

Cases of KFD were reviewed regarding clinical data and histopathological findings. The clinical data included age, sex, presentation and outcome of KFD, while the histopathological findings included histopathologic phases of KFD (using Kuo classification; $1995)^{[5]}$.

As regards histopathological examination, paraffin embedded blocks of KFD cases were cut and stained by Hematoxylin and Eosin (H\&E). Similarly, these cases were also stained by a special stain using Ziehl Neelsen stain for acid fast bacilli. On the other hand, immunohistochemical study was performed using avidin-biotin complex method (ABC) and a panel composed of CD20, CD3 and CD68 with appropriate positive and negative controls.

Regarding the histological picture, cases were classified by using Kuo definition which categorizes KFD into three phases, proliferative, xanthomatous and necrotizing ${ }^{[5]}$. Proliferative phase lesions show sheets of plasmacytoid monocytes, immunoblasts, and a variable number of lymphoid cells, usually without neutrophils. Whereas xanthomatous phase lesions show numerous foamy histiocytes, and finally, necrotizing phase lesions are considered when any degree of coagulative necrosis is present.

\section{Results}

A total number of 1,805 lymph nodes had been sent to our laboratory for histopathological examination in the period between 2001 and 2016. Only 9 cases had been diagnosed as KFD (Table 1), three (33\%) cases were 20 years old and under, five (56\%) cases were in the ages between 20 to 40 years old and only one (11\%) case was above 40 years old. Eight (89\%) cases were females and only one (11\%) case was male. Seven (78\%) cases presented as cervical lymphadenopathy, one (11\%) case as axillary lymphadenopathy and one (11\%) case as bilateral axillary and cervical lymphadenopathy. All these cases showed complete recovery with no recurrence. 
Table 1. Clinical data.

\begin{tabular}{|c|c|c|l|c|c|}
\hline Case N0 & Age & Sex & \multicolumn{1}{|c|}{ Presentation } & Constitutional Signs & $\begin{array}{c}\text { Extra-Nodal and } \\
\text { Cutaneous Manifestation }\end{array}$ \\
\hline 1 & 46 & Female & Left axillary lymphadenopathy & - & - \\
\hline 2 & 29 & Female & Right cervical lymphadenopathy & + & - \\
\hline 3 & 21 & Male & Cervical lymphadenopathy & + & - \\
\hline 4 & 15 & Female & Right cervical lymphadenopathy & - & - \\
\hline 5 & 19 & Female & Cervical lymphadenopathy & + & - \\
\hline 6 & 24 & Female & Left cervical lymphadenopathy & + & - \\
\hline 7 & 38 & Female & History of breast cancer, cervical lymphadenopathy & - \\
\hline 8 & 37 & Female & Bilateral axillary and cervical lymphadenopathy on \& off & + & - \\
\hline 9 & 20 & Female & Cervical lymphadenopathy on \& off & + & - \\
\hline
\end{tabular}

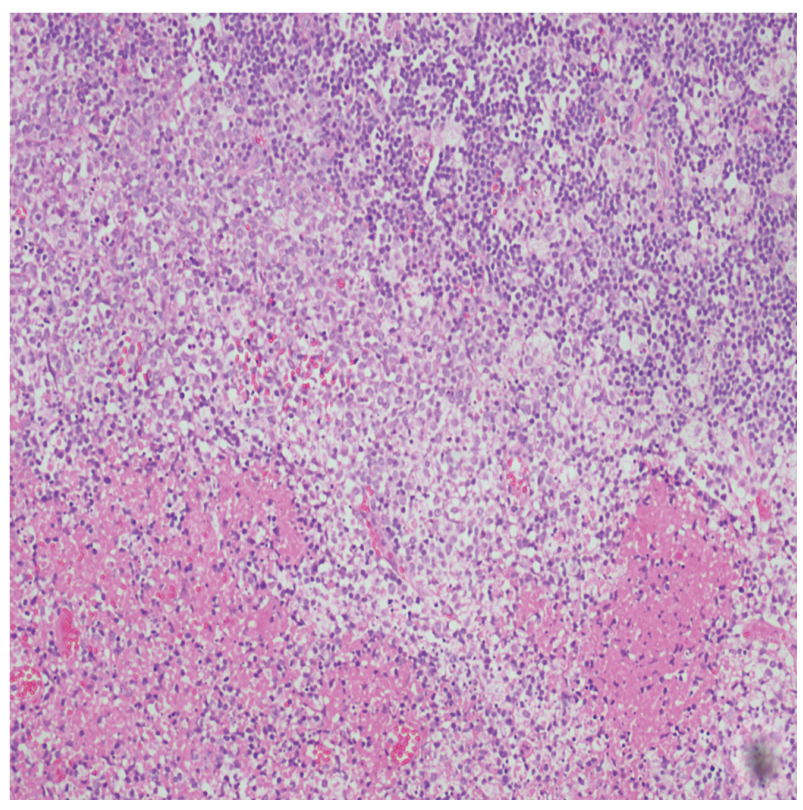

Figure 1a. Kikuchi-Fujimoto disease; lymph node: (a) Areas of necrosis (pink in color, lower part of photo) containing numerous karyorrhectic debris without neutrophils; surrounded by variable amount of histiocytes, and small to medium-sized lymphocytes (upper part of photo); (H\&E stain; power $100 \mathrm{X}$ ).

Constitutional signs were found in seven (77.8\%) cases, which included anorexia, loss of weight, night sweats or high fever. On the contrary, there were no extranodal associated cutaneous manifestations.

The histological picture showed only two histological phases; eight (89\%) cases at necrotic phase and only one (11\%) at proliferative phase; while pure xanthomatous phase was not seen.

The cases at the necrotic phase showed welldefined foci of necrosis, mainly paracortical in location, with partial preservation of the nodal architecture.

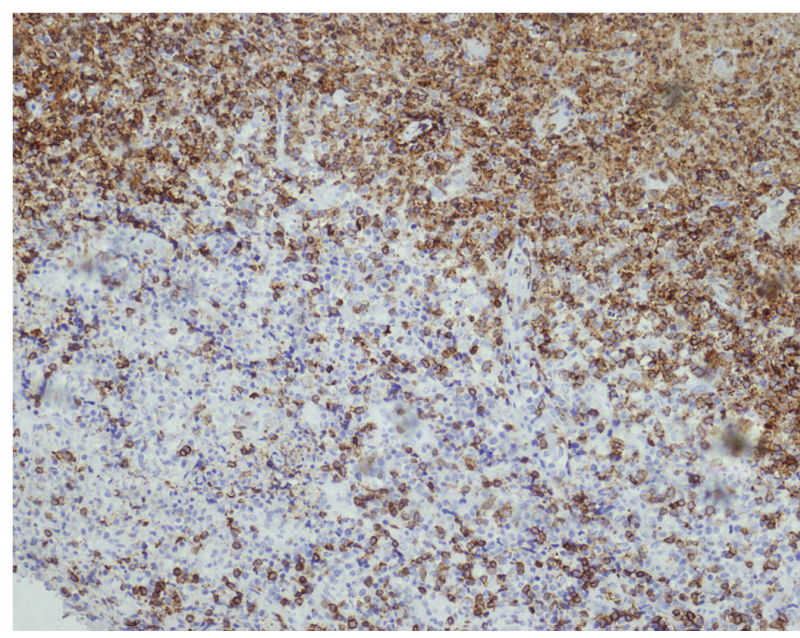

Figure 1b. Kikuchi-Fujimoto disease; lymph node; (b) Intense CD3 positive T-cell infiltrate (right upper corner) around area of necrosis (left lower corner); (immunohistochemistry; power 200 $\mathrm{X})$.

The center of necrosis contained many karyorrhectic debris, but no neutrophils were noted. The periphery of the necrotic zones showed plasmacytoid monocytes admixed with macrophages with twisted nuclei and many immunoblasts (Fig. 1a). By using immunohistochemistry, the cells found around the foci of necrosis showed strong positivity for T-lymphocytic marker (CD3; Fig. 1b) and histiocytic marker (CD68). Few cells were positive for B-lymphocytic marker (CD20). All cases were negative to the Ziehl-Neelsen stain.

The case with proliferative phase was actually a referral case (case number 9). It was composed of many atypical cells (Fig. 2a) and was diagnosed as lymphoma in another hospital. The case was referred to us to confirm diagnosis and to do immunohistochemistry. However, those atypical cells were actually plasmacytoid monocytes and T-cell immunoblasts by 


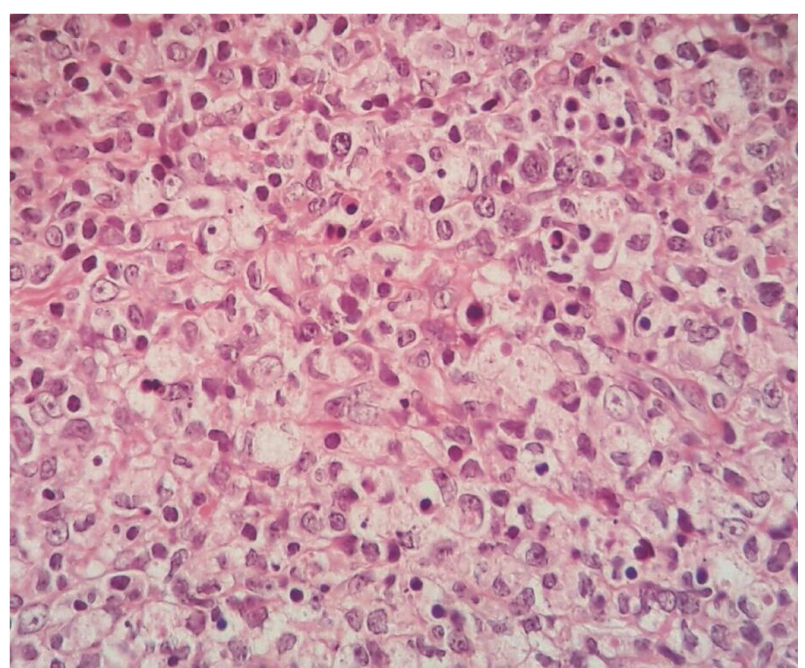

Figure 2a. Kikuchi-Fujimoto disease; lymph node; proliferative phase: (a) many reactive immunoblasts and plasmacytoid monocytes showing atypia that can be mistaken for lymphoma (H\&E stain; power 400 X).

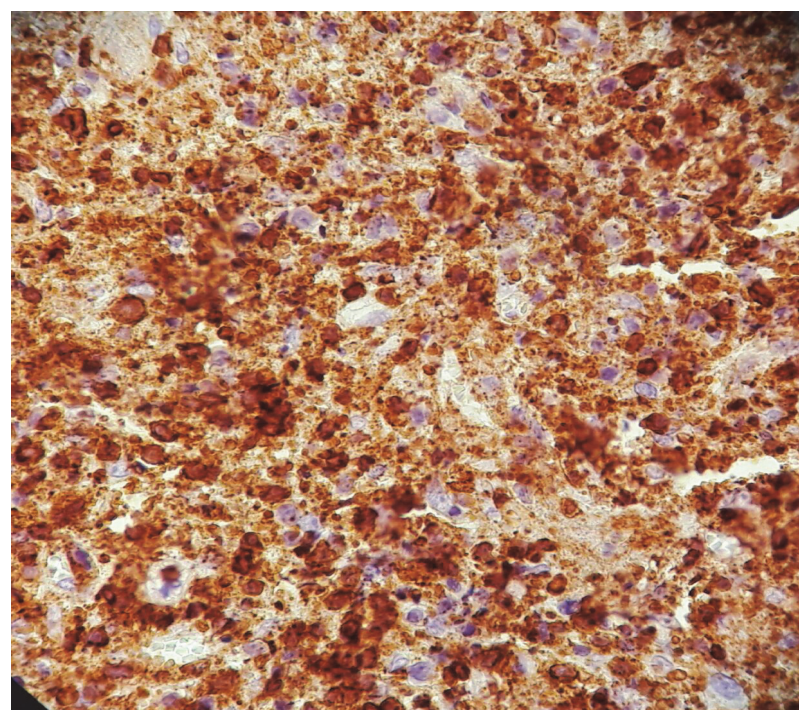

Figure 2c. Kikuchi-Fujimoto disease; Lymph node; proliferative phase: (c) CD3 is positive in T-cell immunoblasts (immunohistochemistry; power 200 X).

immunohistochemistry (Fig. $2 \mathrm{~b}$ and $2 \mathrm{c}$ ), which was consistent with the proliferative phase of KFD.

\section{Discussion}

Kikuchi and Fujimoto initially described this disease in Japan in $1972^{[6]}$. It was fundamentally found in young adult females less than 40 years of age $\mathrm{e}^{[7]}$. KikuchiFujimoto disease may be present with acute or subacute onset developing over several weeks. Enlarged cervical

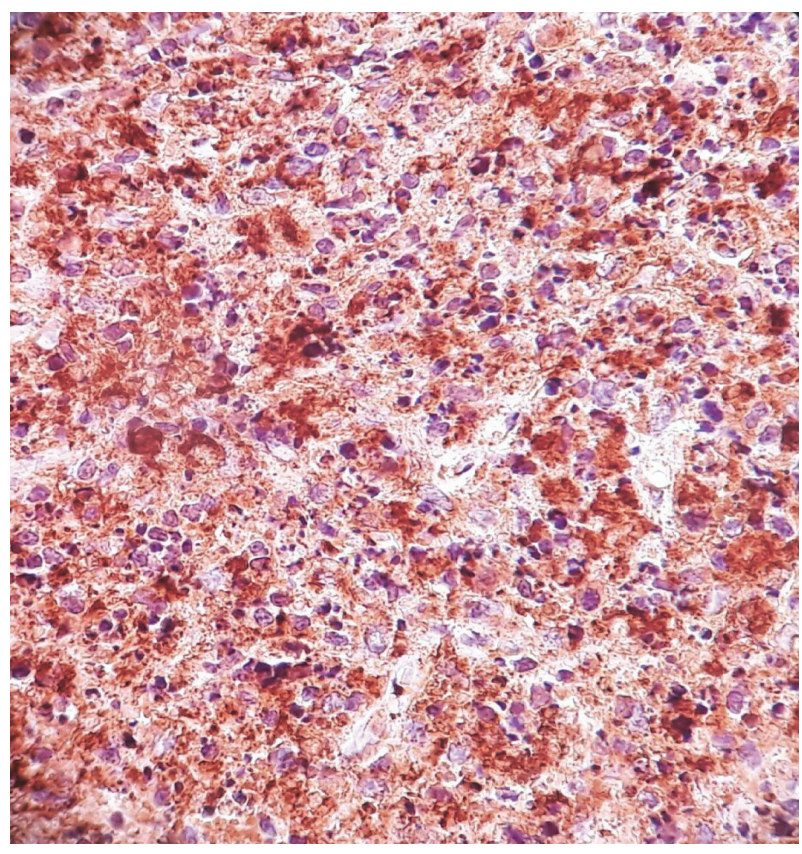

Figure 2b. Kikuchi-Fujimoto disease; Lymph node; proliferative phase: (b) CD68 is positive in plasmacytoid monocytes (immunohistochemistry; power $100 \mathrm{X}$ ).

lymphadenopathy is the major clinical presentation in KFD patients, followed by fever which may be noted in $30 \%$ to $50 \%$ of the affected patients ${ }^{[8]}$. Other less commonly reported manifestations included neutropenia (up to 50\%), lymphocytosis, and atypical lymphocytes found in peripheral smear, liver function impairment, and bone marrow hemophagocytosis ${ }^{[1,3,9]}$.

Kikuchi-Fujimoto disease may clinically and histologically mimic lymphomas and connective tissue disorders associated lymphadenopathies such as systemic lupus erythematosus (SLE), rheumatoid arthritis, and Still's disease; and it may also mimic lymph node involvement by bacterial or viral infections such as infectious mononucleosis, cat scratch disease, herpes simplex, toxoplasmosis, HIV, tuberculosis, and atypical mycobacterial lymphadenitis ${ }^{[7]}$.

As a consequence of the clinical and pathological relationship between KFD and SLE, some authors have proposed that KFD may be a clinical feature or may be an incomplete phase of lupus lymphadenitis ${ }^{[10]}$. However, there are many cases of KFD that had been reported in the absence of SLE${ }^{[11]}$, which may favor the fact that they are two different categories that may often coexist, as it happens with most of the autoimmune disorders in susceptible patients. All our examined patients had no association with SLE. 
Many causative agents of infections have been studiedasapossibleetiologyincluding cytomegalovirus, Epstein-Barr virus, human herpesvirus-6, human immunodeficiency virus, varicella-zoster virus, Yersinia enterocolitica, and toxoplasma ${ }^{[12-14]}$. However, no convincing etiological association between these infectious agents and Kikuchi disease has been settled. In a study done by Dominguez et al. ${ }^{[15]}$. Thirty-two (76\%) out of $42 \mathrm{KFD}$ patients showed highly positive serological titer for HHV6; 10 (24\%) of the cases were positive for HHV6 by using PCR from paraffin blocks; and four (9\%) of the cases were positive by in situ hybridization procedure. In another study, examination of lymphoid tissues obtained from 34 KFD patients by using molecular techniques revealed detection of infectious mononucleosis in only two patients; while all cases showed no relation with $\mathrm{HHV}^{[16]}$.

Kikuchi-Fujimoto disease is mainly diagnosed by histopathological examination of the lymph node. The current study showed that most of the patients in our population were under the age of 40 years $(89 \%)$, most of them were females (89\%) and most of the cases showed constitutional signs (77.8\%). The most common location was cervical region (89\%), only one $(11 \%)$ case was in the axillary region which is an unusual location.

Regarding histologic examination, the current study showed that the necrotic phase was the most common (78\%). By using Ziehl Neelsen special stain, acid fast bacilli organisms could not be detected. Immunohistochemical examination was in favor of the diagnosis of KFD over lymphoma.

Because it may be mistaken for other infective lymphadenitis or lymphoma, identification of KFD is crucial. Patients of KFD have been reported in different regions of the Kingdom of Saudi Arabia ${ }^{[17]}$. The reported KFD clinical picture and pathological pattern of the current study in Saudi patients are similar to those reported in other countries.

The present study established that KFD is a selflimiting disorder without any specific management demand. On the other hand to exclude other differential diagnosis, a follow up is always recommended. And although the sample of patients was limited, our study showed that KFD had marked female prevalence, which is also found in many studies done in western countries. However, on the other hand studies from China paradoxically reported this finding ${ }^{[18]}$.

\section{Conclusion}

Both clinicians and pathologists should be aware of clinical and histological features of the KFD, respectively. Precise light microscopic examination combined with special stains and immunohistochemical studies are useful to reach an accurate diagnosis and to differentiate KFD from malignant lymphomas and infectious lesions, particularly TB.

\section{Conflict of Interest}

The authors have no conflict of interest.

\section{Disclosure}

None of authors received any type of commercial support either in the forms of compensation or financial for this study. The authors have no financial interest in any of the products, devices of drugs mentioned in this article.

\section{Ethical Approval}

Not required.

\section{References}

[1] Lee KY, Yeon YH, Lee BC. Kikuchi-Fujimoto disease with prolonged fever in children. Pediatrics 2004; 114(6): 752756.

[2] Yu HL, Lee SS, Tsai HC, Huang CK, Chen YS, Lin HH, Wann SR, Liu YC, Tseng HH. Clinical manifestations of Kikuchi's disease in Southern Taiwan. J Microbiol Immunol Infect 2005; 38(1): 35-40.

[3] Yasukawa K, Matsumura T, Sato-Matsumura KC, Takahashi T, Fujioka Y, Kobayashi H, Shimizu H. Kikuchi's disease and the skin: case report and review of the literature. $\mathrm{Br} J$ Dermatol 2001;144(4): 885-889.

[4] Pileri SA, Facchetti F, Ascani S, Sabattini E, Poggi S, Piccioli $M$, Rondelli D, Vergoni F, Zinzani PL, Piccaluga PP, Falini $B$, Isaacson PG. Myeloperoxidase expression by histiocytes in Kikuchi's and Kikuchi-like lymphadenopathy. Am J Pathol 2001; 159(3): 915-924.

[5] Kuo TT. Kikuchi's disease (histiocytic necrotizing lymphadenitis). A clinicopathologic study of 79 cases with an analysis of histologic subtypes, immunohistology, and DNA ploidy. Am J Surg Pathol 1995; 19(7): 798-809.

[6] Ruaro B, Sulli A, Alessandri E, Fraternali-Orcioni G, Cutolo M. Kikuchi-Fujimoto disease associated with systemic lupus erythematous: difficult case report and literature review. Lupus 2014; 23(9): 939-944.

[7] Hutchinson CB, Wang E. Kikuchi-Fujimoto disease. Arch Pathol Lab Med 2010; 134(2): 289-293. 
[8] Bosch X, Guilabert A, Miquel R, Campo E. Enigmatic Kikuchi-Fujimoto disease: a comprehensive review. Am J Clin Path 2004; 122(1): 141-152.

[9] Bosch X, Guilabert A. Kikuchi-Fujimoto disease. Orphanet J Rare Dis 2006; 1: 18.

[10] Cramer J, Schmiedel S, Alegre NG, Schafer H, Burchard GD, Merz H. Necrotizing lymphadenitis: Kikuchi-Fujimoto disease alias lupus lymphadenitis? Lupus 2010; 19(1): 8992.

[11] Kucukardali Y, Solmazgul E, Kunter E, Oncul O, Yildirim S, Kaplan M. Kikuchi-Fujimoto disease: analysis of 244 cases. Clin Rheumatol 2007; 26(1): 50-54.

[12] Kapadia V, Robinson BA, Angus HB. Kikuchi's disease presenting as fever of unknown origin. Lancet 1989; 2(8678-8679): 1519-1520.

[13] Sumiyoshi Y, Kikuchi M, Minematu T, Ohshima K, Takeshita M, Minamishima Y. Analysis of herpesvirus genomes in Kikuchi's disease. Virchows Arch 1994; 424(2): 437-440.

[14] Pileri SA, Sabattini E, Costigliola P, Poggi S, Ricchi E, Tumietto F, Chiodo F. Kikuchi's lymphadenitis and HIV infection. AIDS 1991; 5(4): 459-461.

[15] Dominguez DC, Torres ML, Antony S. Is human herpesvirus 6 linked to Kikuchi-Fujimoto disease? The importance of consistent molecular and serologic analysis. South Med J 2003; 96(3): 226-233.

[16] George TI, Jones CD, Zehnder JL, Warnke RA, Dorfman RF. Lack of human herpesvirus 8 and Epstein-Barr virus in Kikuchi's histiocytic necrotizing lymphadenitis. Hum Pathol 2003; 34(2): 130-135.

[17] Al-Nazer MA, Al-Hadad AM, Al-Aithan SA, Al-Salem AH, Al-Faraj AA, Al-Saeed HH. Kukuchi [corrected] Fujimito [corrected] disease. Saudi Med J 2002; 23(4): 405-408.

[18] Lin HC, Su CY, Huang CC, Hwang CF, Chien CY. Kikuchi's disease: a review and analysis of 61 cases. Otolaryngol Head Neck Surg 2003; 128(5): 650-653. 


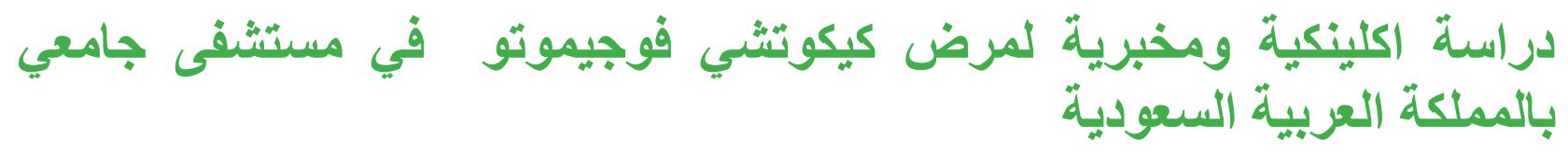

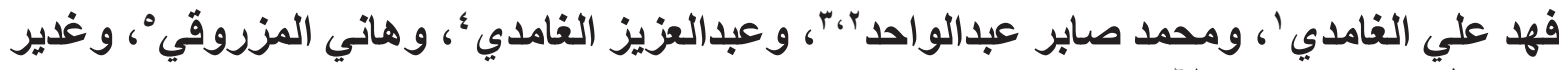

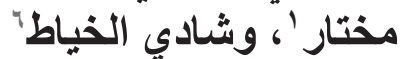

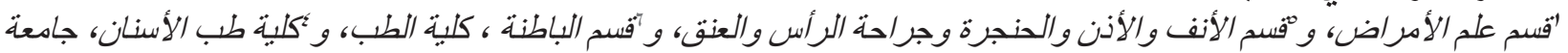

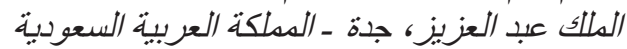

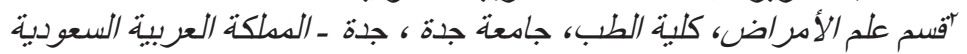

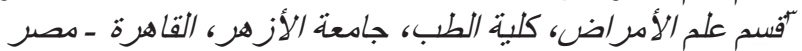

المستخلص. مرض كيكوتشي فوجيموتو يعد مرض ذا عو اقب حميدة بسبب غالبا تضخم في الغدد الليمفاوية والتي قد تشابه

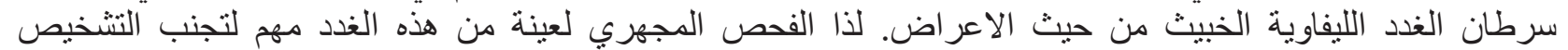

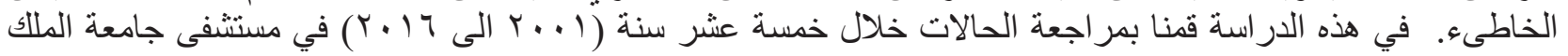

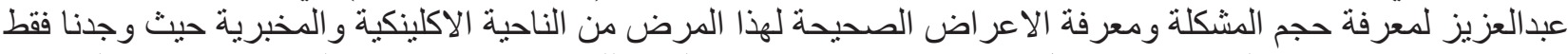

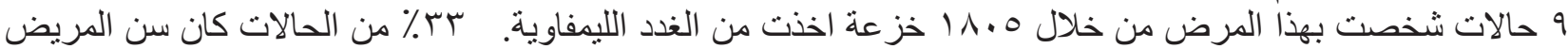

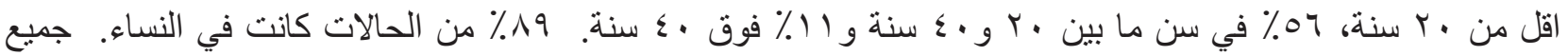
الحالات المرضى تم شفائهم بدون اي عو اقب. من الناحية المخبرية كان هناك ثناثة اشكال. 